\title{
INTERNAÇÕES POR ACIDENTES DE TRANSPORTE TERRESTRE ENVOLVENDO MOTOCICLETAS
}

\section{Hospitalizations due to road traffic crashes involving motorcycles \\ Ingresos hospitalarios por accidentes de transporte terrestre con bicicletas}

Ulicélia Nascimento de Azevedo

Universidade Federal do Rio Grande do Norte - UFRN - Natal (RN) - Brasil

Ana Patrícia de Queiroz Medeiros Dantas

Universidade Federal do Rio Grande do Norte - UFRN - Natal (RN) - Brasil

Marilane Vilela Marques

Secretaria Municipal de Saúde de Natal - SMS - Natal (RN) - Brasil

Ana Edimilda Amador

Universidade Federal do Rio Grande do Norte - UFRN - Natal (RN) - Brasil

Aryelly Dayane da Silva Nunes

Universidade Federal do Rio Grande do Norte - UFRN - Natal (RN) - Brasil

Yonara Monique da Costa Oliveira

Universidade Federal do Rio Grande do Norte - UFRN - Natal (RN) - Brasil

Isabelle Ribeiro Barbosa

Universidade Federal do Rio Grande do Norte - UFRN - Natal (RN) - Brasil

\section{RESUMO}

Objetivo: Analisar as internações por acidentes de transporte terrestre envolvendo motocicletas. Métodos: Estudo descritivo sobre internações por acidentes de transporte terrestre (IATT) envolvendo motocicletas com residentes do estado do Rio Grande do Norte, Brasil, no período de 2008 a 2016, pagas pelo SUS, a partir de dados do Sistema de Informação Hospitalar do Sistema Único de Saúde (SIH/SUS). Analisaram-se as taxas de internações por sexo, faixa etária e região de saúde, além da proporção dos gastos devido à internação. Resultados: No período analisado ocorreram 15.233 IATT envolvendo motociclistas, com coeficiente de internação de 4,38/1000 habitantes. Observouse maior coeficiente de internação para o sexo masculino (6:1). Destacam-se as faixas etárias de 20-34 anos e acima de 70 anos. A Região Metropolitana registrou o maior número de internações por acidentes com motos $(n=4.260)$, porém a Regional de Santa Cruz apresentou a maior proporção de acidentes de motos relacionada às IATT (75\%). O estado assumiu o gasto de R $\$ 17.916 .327,90$ com a internação por esses acidentes, o que representa 66,16\% dos gastos com IATT. Conclusão: Encontrou-se elevada proporção de internações por acidentes com motocicletas no estado do Rio Grande do Norte em relação às internações por acidentes de transporte terrestre, acometendo principalmente jovens do sexo masculino, com aumento de gastos nessas internações.

Descritores: Acidentes de trânsito; Motocicletas; Hospitalização; Causas Externas.

\section{ABSTRACT}

Objective: To analyze hospitalizations due to road traffic crashes involving motorcycles. Methods: A descriptive study of SUS-funded hospitalizations due to road traffic crashes (RTC) involving motorcycles in people living in the State of Rio Grande do Norte, Brazil, from 2008 to 2016. Data were obtained from the Hospital Information System of the Unified Health System (SIH/SUS). Hospitalization rates were stratified by gender, age group and health region. The proportion of hospitalization costs was also analyzed. Results: There were 15,233 $R T C$ involving motorcyclists in the period analyzed, with a hospitalization coefficient of 4.38/1000 inhabitants. The hospitalization coefficient was higher among men (6:1). The most prevalent age groups were 20-34 years and over 70 years. The Metropolitan Region recorded the highest number of hospitalizations due to motorcycle accidents $(n=4,260)$, but the Santa Cruz Region presented the highest proportion of motorcycle accidents in relation to the RTC (75\%). The State Government spent R\$17,916,327.90 on hospitalizations due to these accidents, which represents $66.16 \%$ of RTC expenses. Conclusion: There was a high proportion of hospitalizations due to motorcycle crashes in the state of Rio Grande do Norte in relation to road traffic crashes. The crashes involved mostly young men, with increased expenses due to these hospitalizations.

Descriptors: Accidents, Traffic; Motorcycles; Hospitalization; External Causes. 


\section{RESUMEN}

Objetivo: Analizar los ingresos hospitalarios por accidentes de transporte terrestre con motocicletas. Métodos: Estudio descriptivo sobre los ingresos hospitalarios por accidentes de transporte terrestre (IATT) con motocicletas con residentes del estado de Rio Grande do Norte, Brasil, en el periodo entre 2008 y 2016, pagados pelo SUS, a partir de los datos del Sistema de Información del Hospital del Sistema Único de Salud (SIH/SUS). Se analizaron las tasas de ingresos hospitalarios por el sexo, la franja de edad y la región de salud, además de la proporción de los gastos debido el ingreso. Resultados: En el periodo analizado ocurrieron 15.233 IATT con motociclistas, con coeficiente de ingreso hospitalario de 4,38/1000 habitantes. Se observó un mayor coeficiente de ingreso hospitalario para el sexo masculino (6:1). Se destacaron las franjas de edad entre 20-34 años y después de los 70 años. La región metropolitana ha registrado el mayor número de ingresos hospitalarios por accidentes con motos $(n=4.260)$ pero la Regional de Santa Cruz presentó la mayor proporción de accidentes con motos relacionada con los IATT (75\%). El estado asumió el gasto de $R \$ 17.916 .327,90$ con los ingresos por eses accidentes lo que representa el 66,16\% de los gastos con los IATT. Conclusión: Se encontró elevada proporción de ingresos hospitalarios por accidentes con motocicletas en el estado del Rio Grande do Norte respecto los ingresos hospitalarios por accidentes de transporte terrestre que acometen principalmente a los jóvenes del sexo masculino con el aumento de gastos para los ingresos hospitalarios.

Descriptores: Accidentes de Tránsito; Motocicletas; Hospitalización; Causas Externas.

\section{INTRODUÇÃO}

As causas externas ganharam relevância nas últimas décadas pelo importante impacto que esses agravos geram na atenção e na assistência em saúde, elevando os custos nos setores e nos níveis assistenciais envolvidos, além de apresentar grande relevância na morbidade e na mortalidade da população ${ }^{(1,2)}$. Os serviços que mais se sobrecarregam pelas altas demandas decorrentes dos acidentes e violências são principalmente os serviços públicos de emergência, de assistência especializada, de reabilitação física, psicológica e de assistência social ${ }^{(3)}$.

A Classificação Internacional de Doenças em sua $10^{\mathrm{a}}$ revisão(4) ${ }^{(4)}$ classifica acidente de trânsito como aquele que envolve veículo transitando em via pública. Esses acidentes acometem principalmente com pessoas nas faixas etárias mais jovens e produtivas da população, com enormes repercussões econômicas, sociais e emocionais ${ }^{(5)}$.

A projeção é de que, em 2020, o número de óbitos por acidentes de transporte terrestre (ATT) seja de 2,4 milhões e represente a sexta causa de morte no mundo. O Brasil ocupa o quinto lugar no ranking dos países com maior número de mortes por acidentes de trânsito, atrás apenas da Índia, da China, dos Estados Unidos e da Rússia. Uma das características do padrão epidemiológico dos ATT no Brasil tem sido o aumento dos acidentes por motocicletas, a sobremortalidade masculina e a maior incidência na faixa etária dos adultos jovens, de 20 a $39 \operatorname{anos}^{(6)}$.

Em países emergentes e em desenvolvimento econômico, como é o caso do Brasil, houve um expressivo crescimento da frota de motocicletas, representando entre 50,0 e $70,0 \%$ da frota total de veículos ${ }^{(7)}$. A causalidade dos acidentes envolvendo os motociclistas é multifatorial, destacando-se a vulnerabilidade devido ao tipo de veículo, o aumento vertiginoso da frota, a segurança viária veicular, o comportamento de risco, além da utilização crescente desse veículo como instrumento de trabalho sem uma abordagem voltada para a segurança laboral ${ }^{(8)}$.

O Ministério da Saúde (MS) alertou para o impacto negativo desses acidentes sobre a saúde da população brasileira, a perda de anos de vida livres de incapacidade, a redução da expectativa de vida dos adolescentes e jovens, além dos altos custos sociais e econômicos impostos ao sistema de saúde e previdenciário ${ }^{(9)}$. Em 2014, no Brasil, foram registradas 96.292 internações de motociclistas, resultando um dispêndio de 26 milhões de reais, o que representou $52 \%$ dos gastos com internação de vítimas de ATT no Sistema Único de Saúde (SUS) ${ }^{(8)}$.

Apesar da implantação do Código Nacional de Trânsito, em 1998, com o estabelecimento de normas legais para punir os infratores e da Lei $\mathrm{n}^{\circ} 11.705$, de 19 de junho de 2008, conhecida como Lei Seca, que tem entre suas finalidades estabelecer alcoolemia zero e impor penalidades severas ao condutor que dirigir sob efeito do álcool, os acidentes de trânsito ainda representam uma importante causa de mortalidade precoce e evitável no Brasil ${ }^{(7,10-12)}$.

Em março de 2010, a Organização das Nações Unidas proclamou o período 2011 a 2020 como a década de ação pela segurança no trânsito. Nesse contexto, o Brasil constituiu a Política Nacional de Redução da Morbimortalidade por Acidentes e Violência ${ }^{(13)}$, com um plano de ação voltado para cinco pilares de intervenção: fortalecimento da gestão, investimento em infraestrutura viária, segurança veicular, comportamento e segurança dos usuários do trânsito, e atendimento pré-hospitalar e hospitalar ao trauma ${ }^{(9)}$.

Pelo exposto, fica evidente a necessidade de investigação sobre o tema. Dentre as fontes de informações mais utilizadas no monitoramento da morbimortalidade destes eventos no Brasil, cita-se o Sistema de Informações Hospitalares do SUS (SIH/SUS) ${ }^{(14)}$, que fornece informações sobre a morbidade hospitalar e os gastos com esses acidentes, dentre outras. Essas informações poderão auxiliar na execução de estudos futuros e servir de material de consulta para implementação de ações 
intersetoriais que envolvam ações no âmbito da gestão dos serviços de saúde e de promoção da saúde. Essas ações de prevenção de acidentes, principalmente dos que envolvem motocicletas, de promoção da saúde e de cultura da paz são indispensáveis para a redução nos custos com internação e reabilitação, principalmente nos serviços públicos de saúde.

O objetivo do presente estudo foi analisar as internações por acidentes de transporte terrestre envolvendo motocicletas.

\section{MÉTODOS}

Trata-se de estudo descritivo sobre as internações por acidentes de transporte terrestre (IATT) envolvendo motocicletas, realizado com residentes do estado do Rio Grande do Norte (RN), ocorridas no período de 2008 a 2016 cujas internações hospitalares foram pagas pelo SUS. A escolha do período justifica-se pela possibilidade de realizar a análise temporal ao longo de nove anos, além de diminuir a possibilidade de erro por retardo na entrada dos dados.

Em 2016, o estado do Rio Grande do Norte apresentava população estimada de 3.474.998 habitantes, distribuída entre 167 municípios e 8 regiões de saúde ${ }^{(15)}$. Nesse mesmo ano, a rede hospitalar de gestão estadual era constituída por 24 hospitais que disponibilizavam 1.589 leitos, distribuídos entre as oito regiões de saúde do estado da seguinte forma: 8 na região Metropolitana, 3 na região de São José de Mipibu, 3 na região de Mossoró, 3 na região de Caicó, 3 na região de Açu, 1 na região de João Câmara, 2 na região de Santa Cruz e 1 na região de Pau dos Ferros ${ }^{(16)}$.

Coletaram-se os dados do Sistema de Informações Hospitalares do Sistema Único de Saúde (SIH/SUS), disponibilizado no sítio eletrônico do Departamento de Informática do SUS (DATASUS). Adotou-se o diagnóstico principal das internações registrado no SIH/SUS como acidentes com transporte terrestre envolvendo motocicletas (V20-V29) como causa de internação, de acordo com a CID-10. Consideraram-se as demais internações por causas externas e as internações por todas as causas para as análises deste estudo.

Observou-se o perfil das internações por faixa etária (0-19 anos; 20-34 anos; 35-49 anos; 50-69 anos; >70 anos), por sexo (masculino e feminino) e por região de saúde da ocorrência do acidente no estado.

Calculou-se o coeficiente de IATT envolvendo motociclistas (número de IATT envolvendo motociclistas de pacientes residentes no Rio Grande do Norte dividido pela população do RN, multiplicado por mil habitantes), o coeficiente de IATT (número de IATT de pacientes residentes no RN dividido pela população do RN, multiplicado por mil habitantes), o coeficiente de internações por causas externas (número de internações por causas externas em residentes no RN dividido pela população do RN, multiplicado por mil habitantes), o coeficiente de internações por sexo (número de internações por causas externas, por IATT e por IATT envolvendo motociclistas, de acordo com o sexo do paciente residente no RN dividido pela população por sexo do RN, multiplicado por mil habitantes) e o coeficiente de IATT por faixa etária (número de IATT envolvendo motociclistas por faixa etária em residentes no RN dividido pela população por faixa etária do RN, multiplicado por mil habitantes).

Calcularam-se as seguintes proporções: a) proporção de IATT envolvendo motociclistas (número de IATT envolvendo motociclistas de pacientes residentes no RN dividido pelo número de IATT de pacientes residentes no RN, multiplicado por 100) e b) proporção de IATT (número de IATT de pacientes residentes no RN dividido pelo número de internações por causas externas de pacientes residentes no RN, multiplicado 100).

Calcularam-se os valores totais das internações por IATT, em reais (R\$), segundo a proporção de gastos anuais com as IATT envolvendo motociclistas (gasto com IATT envolvendo motociclistas de pacientes residentes no RN dividido pelo total de gastos com IATT de pacientes residentes no RN), a proporção de gastos com IATT dentre as causas externas (gasto com IATT de pacientes residentes no RN dividido pelo total de gastos com por causas externas de pacientes residentes no RN), e a proporção de gastos por ATT dentre o total de internações (gasto com IATT de pacientes residentes no RN dividido pelo total de gastos com internações de pacientes residentes no RN), por triênios no período de 2008 a 2016. Analisaram-se todos os coeficientes e proporções por períodos de três anos: 2008-2010; 2011-2013; 2014-2016.

Realizou-se o cálculo a partir da autorização de internação hospitalar (AIH) do SIH/SUS, que consiste em um resumo da alta hospitalar preenchido pelos hospitais prestadores do SUS para recebimento das internações por eles realizadas. Coletaramse os dados referente à população no sítio eletrônico do Instituto Brasileiro de Geografia e Estatística ${ }^{(15)}$.

A presente pesquisa utilizou dados secundários disponíveis em sites oficiais do Ministério da Saúde do Brasil, sem identificação de sujeitos, sendo dispensado de apreciação em comitê de ética em pesquisa, em conformidade com a Resolução 466/2012 do Conselho Nacional de Saúde.

\section{RESULTADOS}

No período de 2008 a 2016 computaram-se 99.369 internações com coeficiente de internação por causas externas de 28,60/1000 mil habitantes no estado do Rio Grande do Norte (RN), sendo o triênio de 2011 a 2013 o que apresentou maior coeficiente de internação por causas externas (10,23/1000 mil habitantes), contabilizando 35.555 internações.

Quanto ao coeficiente de internação por acidente de transporte terrestre, contabilizou-se, no período de 2008 a 2016 , 22.197 internações, com coeficiente de 6,39/1000 mil habitantes, destacando-se o período de 2011 a 2013 em que houve 8.546 internações, com coeficiente de 2,46/1000 mil habitantes, o mais elevado de todo o período de estudo. 
Para o coeficiente de internação por motociclista traumatizado em acidente de transporte (V20-V29), no período de 2008 a 2016, no estado do Rio Grande do Norte, observou-se 15.233 autorizações de internações e coeficiente de internação de 4,38/1000 habitantes. Avaliando este dado por triênios, observou-se que o período 2011 a 2013 apresentou o maior número de internações $(n=6.133)$ e coeficiente $1,76 / 1000$ habitantes (Figura 1). Observou-se que 22,3\% ( $n=22197)$ das internações por causas externas envolveram ATT, e que 68,6\% $(\mathrm{n}=15233)$ das internações por ATT envolveram motociclistas traumatizados.

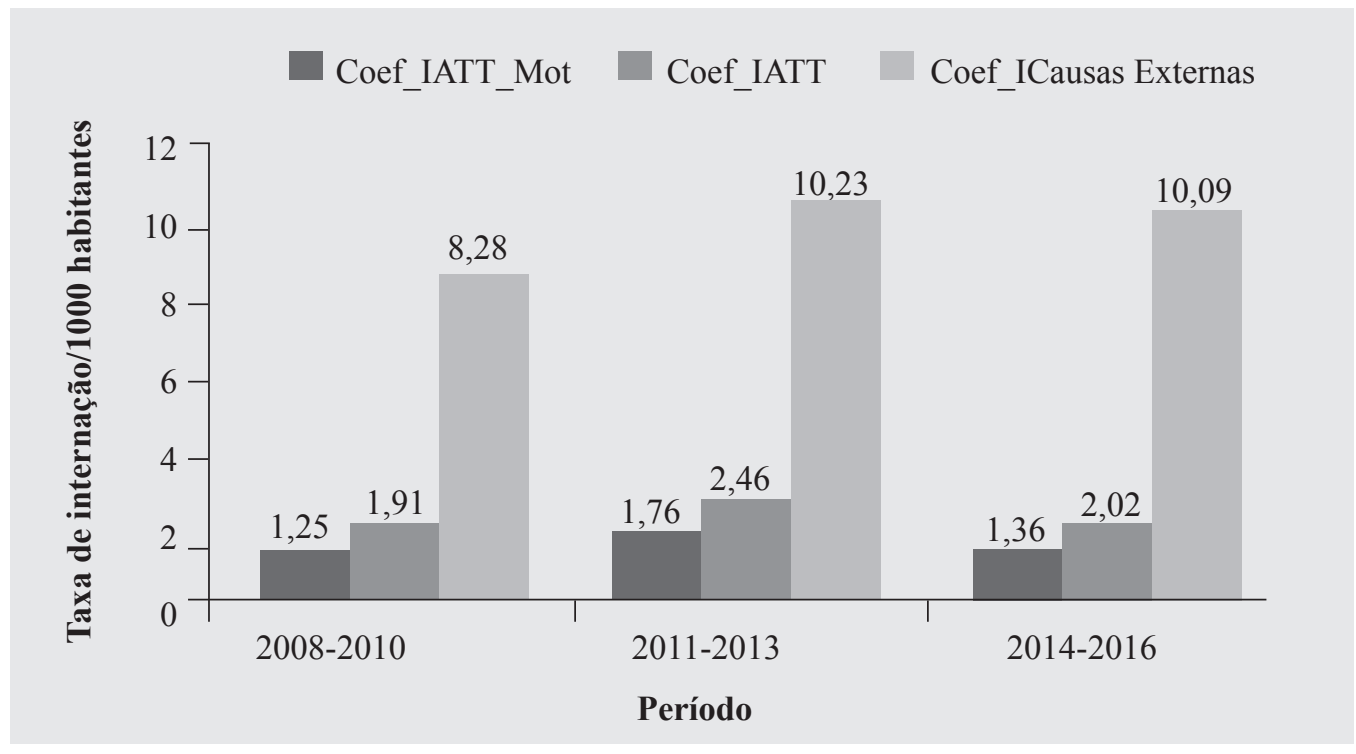

Figura 1 - Coeficiente de internação por causa externa (Coef_ICausas Externas), por acidente de transporte terrestre (Coef IATT) e por acidente envolvendo motociclistas (Coef_IATT_Mot) por triênios no estado do Rio Grande do Norte, Brasil, 2008 a 2016.

Para o coeficiente de internação por causa externa, IATT e IATT envolvendo motociclista, segundo o sexo, no período de 2008 a 2016, observaram-se coeficientes mais elevados para o sexo masculino em todos os anos da série, com destaque para o ano de 2011, apresentando para as causas externas coeficiente de internação de 5,32/1000 homens, coeficiente de IATT de 1,60/1000 homens e IATT envolvendo motociclista de 1,17/1000 homens (Tabela I).

Tabela I - Coeficiente de internação por causas externas, por acidente de transporte terrestre e acidentes envolvendo motociclistas, segundo sexo e por ano, no estado do Rio Grande do Norte, Brasil, 2008 a 2016.

\begin{tabular}{ccccccc}
\hline \multirow{2}{*}{$\begin{array}{c}\text { Ano } \\
\text { atendimento }\end{array}$} & \multicolumn{2}{c}{$\begin{array}{c}\text { Internação por } \\
\text { causas externas }\end{array}$} & \multicolumn{2}{c}{$\begin{array}{c}\text { Internação acidente de } \\
\text { transporte terrestre }\end{array}$} & \multicolumn{2}{c}{$\begin{array}{c}\text { Internação acidente de } \\
\text { transporte terrestre-moto }\end{array}$} \\
\cline { 2 - 7 } & Masc & Fem & Masc & Fem & Masc & Fem \\
\hline $\mathbf{2 0 0 8}$ & 3,92 & 1,42 & 0,90 & 0,19 & 0,58 & 0,09 \\
$\mathbf{2 0 0 9}$ & 4,20 & 1,56 & 1,01 & 0,24 & 0,70 & 0,11 \\
$\mathbf{2 0 1 0}$ & 5,08 & 1,74 & 1,55 & 0,32 & 1,12 & 0,18 \\
$\mathbf{2 0 1 1}$ & 5,32 & 1,92 & 1,60 & 0,34 & 1,17 & 0,19 \\
$\mathbf{2 0 1 2}$ & 5,19 & 1,83 & 1,49 & 0,31 & 1,12 & 0,17 \\
$\mathbf{2 0 1 3}$ & 5,28 & 1,90 & 1,23 & 0,25 & 0,95 & 0,14 \\
$\mathbf{2 0 1 4}$ & 5,22 & 2,02 & 1,21 & 0,25 & 0,87 & 0,14 \\
$\mathbf{2 0 1 5}$ & 4,87 & 1,93 & 1,13 & 0,26 & 0,79 & 0,13 \\
$\mathbf{2 0 1 6}$ & 4,54 & 1,93 & 0,86 & 0,22 & 0,61 & 0,12 \\
\hline
\end{tabular}

Masc: masculino; Fem: feminino 
Ao avaliar os coeficientes de causas externas, IATT e IATT por motociclistas, em relação ao sexo e à faixa etária, no período de 2008 a 2016, constatou-se um coeficiente de internação por causa externa mais elevado para o sexo feminino na faixa etária de 70 anos ou mais (8,89/1000 habitantes do sexo feminino com 70 anos ou mais).

Para IATT, registraram-se os maiores coeficientes para o sexo masculino em todas as faixas etárias, com maior destaque para a faixa de 20 a 34 anos (2,01/1000 homens) e de 35 a 50 anos (1,53/1000 homens). Para IATT envolvendo motociclista observou-se maior coeficiente para homens na faixa etária de 20-34 anos, sendo de 1,60/1000 homens (Figura 2).

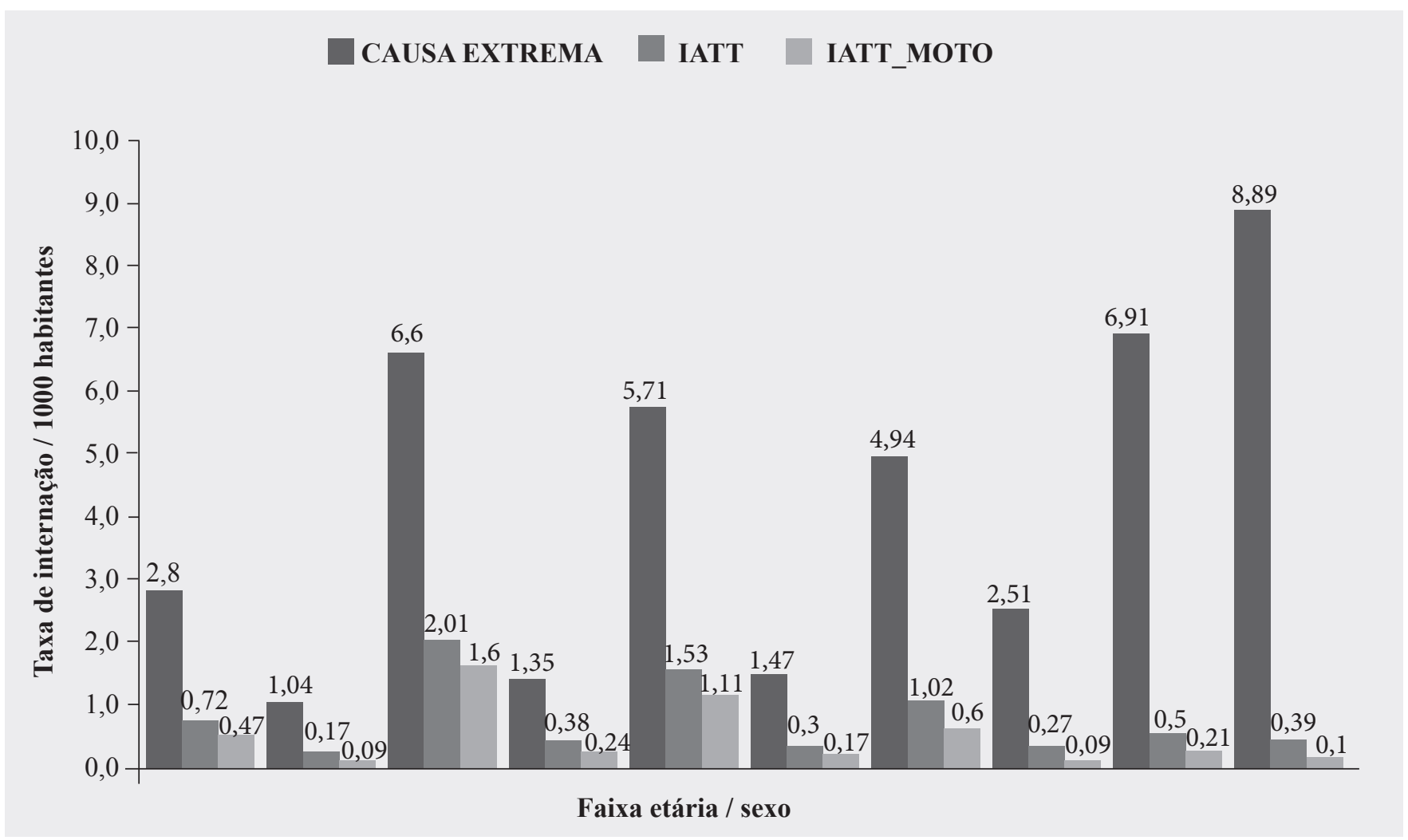

Figura 2 - Coeficiente de internação por causa externa, por acidente de transporte terrestre (IATT), por acidente envolvendo motociclistas (IATT_moto), por sexo e faixa etária, no estado do Rio Grande do Norte, Brasil, 2008 a 2016.

Observa-se que na região Metropolitana registrou-se o maior número de autorizações de internação hospitalar por causas externas, correspondendo a 40,4\% $(n=40.185)$ das internações hospitalares por causas externas do estado, seguida pelas regiões de Mossoró, com 12.688 internações (12,8\%), e Caicó, com 11.986 internações (12,1\%). Para internação hospitalar por IATT envolvendo motociclistas, observou-se maior número de internações na região Metropolitana, com 4.260 (28\%), seguida pela região de Mossoró, com 3.468 (22,8\%), e a região de São José de Mipibu, com 1.617 (10,6\%).

A proporção de internação por IATT envolvendo motociclista para cada região do Rio Grande do Norte, no período de 2008 a 2016, apresentou-se maior na região de Santa Cruz (75\%), seguido da região de Caicó (74,5\%) e de São José de Mipibu (73,1\%). Ao se avaliar comparativamente os triênios para todas as regiões analisadas, percebe-se que houve aumento da proporção de IATT envolvendo motociclistas no período 2011-2013 quando comparado ao período compreendido entre 20082010; contudo, houve tendência de queda nessa proporção no triênio 2014-2016 (Tabela II). 
Tabela II - Proporção de internação por acidentes envolvendo motociclista em relação às internações por acidente de transporte terrestre, por região de saúde, no estado do Rio Grande do Norte, Brasil, nos triênios 2008-2010, 2011-2013 e 2014-2016.

\begin{tabular}{|c|c|c|c|c|c|c|c|c|c|}
\hline \multicolumn{2}{|c|}{ Região de Saúde } & $\begin{array}{c}\text { Internações } \\
\text { 2008-2010 }\end{array}$ & $\begin{array}{c}\text { \% IATT } \\
\text { Moto/ } \\
\text { IATT }\end{array}$ & $\begin{array}{c}\text { Internações } \\
2011-2013\end{array}$ & $\begin{array}{l}\text { \% IATT } \\
\text { Moto/ } \\
\text { IATT }\end{array}$ & $\begin{array}{c}\text { Internações } \\
\text { 2014-2016 }\end{array}$ & $\begin{array}{c}\text { \% IATT } \\
\text { Moto/ } \\
\text { IATT }\end{array}$ & $\begin{array}{c}\text { Total } \\
\text { interno }\end{array}$ & $\begin{array}{c}\% \text { Total } \\
\text { IATT } \\
\text { Moto/IATT }\end{array}$ \\
\hline \multirow[b]{2}{*}{$\begin{array}{l}\text { São José de } \\
\text { Mipibu }\end{array}$} & IATT & 683 & \multirow[b]{2}{*}{70,72} & 831 & \multirow[b]{2}{*}{75,33} & 698 & \multirow[b]{2}{*}{72,78} & 2212 & \multirow[b]{2}{*}{73,10} \\
\hline & $\begin{array}{l}\text { IATT }^{-} \\
\text {Moto }\end{array}$ & 483 & & 626 & & 508 & & 1617 & \\
\hline \multirow[b]{2}{*}{ Mossoró } & IATT & 1458 & \multirow[b]{2}{*}{61,66} & 2168 & \multirow[b]{2}{*}{69,33} & 1921 & \multirow[b]{2}{*}{55,49} & 5547 & \multirow[b]{2}{*}{62,52} \\
\hline & $\begin{array}{l}\text { IATT }_{-} \\
\text {Moto }\end{array}$ & 899 & & 1503 & & 1066 & & 3468 & \\
\hline \multirow[b]{2}{*}{ João Câmara } & IATT & 557 & \multirow[b]{2}{*}{67,68} & 791 & \multirow[b]{2}{*}{74,59} & 690 & \multirow[b]{2}{*}{74,78} & 2038 & \multirow[b]{2}{*}{72,77} \\
\hline & $\begin{array}{l}\text { IATT }_{-} \\
\text {Moto }\end{array}$ & 377 & & 590 & & 516 & & 1483 & \\
\hline \multirow[b]{2}{*}{ Caicó } & IATT & 448 & \multirow[b]{2}{*}{71,88} & 491 & \multirow[b]{2}{*}{75,76} & 444 & \multirow[b]{2}{*}{75,90} & 1383 & \multirow[b]{2}{*}{74,55} \\
\hline & $\begin{array}{l}\text { IATT }^{-} \\
\text {Moto }\end{array}$ & 322 & & 372 & & 337 & & 1031 & \\
\hline \multirow[b]{2}{*}{ Santa Cruz } & IATT & 409 & \multirow[b]{2}{*}{72,13} & 509 & \multirow[b]{2}{*}{77,01} & 433 & \multirow[b]{2}{*}{75,52} & 1351 & \multirow[b]{2}{*}{75,06} \\
\hline & $\begin{array}{l}\text { IATT }_{-} \\
\text {Moto }\end{array}$ & 295 & & 392 & & 327 & & 1014 & \\
\hline \multirow[b]{2}{*}{ Pau dos Ferros } & IATT & 614 & \multirow[b]{2}{*}{70,36} & 722 & \multirow[b]{2}{*}{75,62} & 546 & \multirow[b]{2}{*}{77,47} & 1882 & \multirow[b]{2}{*}{74,44} \\
\hline & $\begin{array}{l}\text { IATT }_{-} \\
\text {Moto }\end{array}$ & 432 & & 546 & & 423 & & 1401 & \\
\hline \multirow[b]{2}{*}{ Metropolitana } & IATT & 2076 & \multirow[b]{2}{*}{61,08} & 2542 & \multirow[b]{2}{*}{68,73} & 1837 & \multirow[b]{2}{*}{67,77} & 6455 & \\
\hline & $\begin{array}{l}\text { IATT }^{-} \\
\text {Moto }\end{array}$ & 1268 & & 1747 & & 1245 & & 4260 & 66,00 \\
\hline & IATT & 393 & & 492 & & 444 & & 1329 & \\
\hline Açu & $\begin{array}{l}\text { IATT }_{-} \\
\text {Moto }\end{array}$ & 283 & 72,01 & 357 & 72,56 & 319 & 71,85 & 959 & 72,16 \\
\hline & IATT & 6638 & & 8546 & & 7013 & & 22197 & \\
\hline Total & $\begin{array}{l}\text { IATT_ } \\
\text { Moto }\end{array}$ & 4359 & 65,67 & 6133 & 71,76 & 4741 & 67,60 & 15233 & 68,63 \\
\hline
\end{tabular}

Fonte: DATASUS, Sistema de Internação Hospitalar, 2017.

Legenda: IATT: Número de Internações por acidentes de transporte terrestre. IATT_Moto: Número de Internações por acidentes de transporte terrestre envolvendo motociclista. \% (IATT_Moto/IATT): proporção de IATT envolvendo motociclistas; intern: internações.

Quanto aos gastos anuais com as IATT envolvendo motociclistas, IATT e internações por causas externas de pacientes residentes no estado, observa-se um gasto de $\mathrm{R} \$ 1.570 .661 .383,70$ reais com internações gerais. Deste total, os gastos com internações por causas externas, por IATT e por IATT envolvendo motociclistas perfizeram o montante de 109.718.203,42 reais, 27.080.848,59 reais e 17.916.327,90 reais respectivamente. Proporcionalmente, os gastos com IATT envolvendo motociclista correspondem a 66,16\% dos gastos com as IATT e 16,32\% dos gastos com internações relacionadas às causas externas.

Ao avaliar isoladamente os triênios, observou-se que houve evolução nos gastos gerais de internação e de internação por causas externas entre os períodos de 2008-2010, 2011-2013 e 2014-2016, porém, em relação aos gastos com IATT e IATT_ Moto, houve aumento no triênio 2011-2013, seguido por uma redução no triênio 2014-2016 (Tabela III). 
Tabela III - Proporção de gastos com internação por acidente de transporte terrestre, por IATT_Motociclista por IATT, IATT por causa externa e IATT por internações gerais no estado do Rio Grande do Norte, 2008 a 2016.

\begin{tabular}{cccccccc}
\hline \multirow{2}{*}{ ANO } & $\begin{array}{c}\text { GASTOS } \\
\text { IATT_Moto }\end{array}$ & $\begin{array}{c}\text { GASTOS } \\
\text { IATT }\end{array}$ & $\begin{array}{c}\text { GASTOS } \\
\text { ICE }\end{array}$ & $\begin{array}{c}\text { GASTOS } \\
\text { INT_RN }\end{array}$ & $\begin{array}{c}\text { \% IATT_Moto/ } \\
\text { IATT }\end{array}$ & $\begin{array}{c}\text { \% } \\
\text { IATT/ICE }\end{array}$ & $\begin{array}{c}\text { \% } \\
\text { IATT/INT_RN }\end{array}$ \\
\hline $\mathbf{2 0 0 8}$ & $1.011 .817,51$ & $1.743 .419,85$ & $7.885 .102,22$ & $126.218 .767,93$ & $58,04 \%$ & $22,11 \%$ & $1,38 \%$ \\
$\mathbf{2 0 0 9}$ & $1.301 .378,95$ & $2.174 .782,59$ & $9.347 .473,96$ & $147.840 .025,01$ & $59,84 \%$ & $23,27 \%$ & $1,47 \%$ \\
$\mathbf{2 0 1 0}$ & $2.254 .182,48$ & $3.427 .232,94$ & $11.094 .603,03$ & $161.573 .885,55$ & $65,77 \%$ & $30,89 \%$ & $2,12 \%$ \\
$\mathbf{2 0 0 8 - 2 0 1 0}$ & $4.567 .378,94$ & $7.345 .435,38$ & $28.327 .179,21$ & $435.632 .678,49$ & $62,18 \%$ & $25,93 \%$ & $1,69 \%$ \\
$\mathbf{2 0 1 1}$ & $2.293 .389,41$ & $3.485 .676,49$ & $12.392 .437,52$ & $171.708 .007,30$ & $65,79 \%$ & $28,13 \%$ & $2,03 \%$ \\
$\mathbf{2 0 1 2}$ & $2.675 .426,73$ & $3.910 .178,44$ & $13.376 .090,22$ & $174.349 .239,52$ & $68,42 \%$ & $29,23 \%$ & $2,24 \%$ \\
$\mathbf{2 0 1 3}$ & $2.328 .161,88$ & $3.290 .275,01$ & $13.653 .820,67$ & $188.481 .265,65$ & $70,76 \%$ & $24,10 \%$ & $1,75 \%$ \\
$\mathbf{2 0 1 1 - 2 0 1 3}$ & $7.296 .978,02$ & $10.686 .129,94$ & $39.422 .348,41$ & $534.538 .512,47$ & $68,28 \%$ & $27,11 \%$ & $2,00 \%$ \\
$\mathbf{2 0 1 4}$ & $2.268 .170,30$ & $3.216 .442,18$ & $13.998 .033,14$ & $197.374 .848,98$ & $70,52 \%$ & $22,98 \%$ & $1,63 \%$ \\
$\mathbf{2 0 1 5}$ & $2.189 .304,42$ & $3.282 .051,39$ & $14.541 .618,22$ & $212.521 .040,44$ & $66,71 \%$ & $22,57 \%$ & $1,54 \%$ \\
$\mathbf{2 0 1 6}$ & $1.594 .496,22$ & $2.550 .789,70$ & $13.429 .024,44$ & $190.594 .303,32$ & $62,51 \%$ & $18,99 \%$ & $1,34 \%$ \\
$\mathbf{2 0 1 4 - 2 0 1 6}$ & $6.051 .970,94$ & $9.049 .283,27$ & $41.968 .675,80$ & $600.490 .192,74$ & $66,88 \%$ & $21,56 \%$ & $1,51 \%$ \\
Total & $17.916 .327,90$ & $27.080 .848,59$ & $109.718 .203,42$ & $1.570 .661 .383,70$ & $66,16 \%$ & $24,68 \%$ & $1,72 \%$ \\
\hline
\end{tabular}

Fonte: DATASUS, Sistema de Internação Hospitalar, 2017.

Legenda: IATT: Internações por acidentes de transporte terrestre. IATT_Moto: Internações por acidentes de transporte terrestre envolvendo motociclista. ICE: Internações por causas externas.

INT_RN: Internações no Estado do Rio Grande do Norte. \% (IATT_Moto/IATT): proporção de IATT envolvendo motociclistas. \% (IATT/ ICE): Proporção de IATT dentre as causas externas. \% (IATT/INT_RN): Proporção de IATT dentre todas as internações.

\section{DISCUSSÃO}

Os resultados do presente estudo demonstraram a elevada proporção de internações por acidentes de transporte terrestre envolvendo motociclista no estado do Rio Grande do Norte, afetando principalmente os indivíduos jovens e do sexo masculino, residentes das regiões de Saúde de Santa Cruz, de Caicó e de São José de Mipibu, além dos gastos que superam a média brasileira.

O estudo que avaliou as internações hospitalares por lesões decorrentes de ATT no ano de 2013, no Brasil, apontou que houve 170.805 internações por ATT, com taxas de 85/100 mil habitante, em que as maiores taxas de internação por ATT incluíam indivíduos do sexo masculino e na faixa etária de 20 a 39 anos. Mais da metade das internações por IATT acometeu motociclistas $(51,9 \%)$, seguidos por pedestres $(25,8 \%)^{(17)}$. Esses resultados assemelham-se aos encontrados no Rio Grande do Norte, mostrando que o problema das internações por ATT envolvendo motociclistas no estado não é uma exceção no contexto nacional.

O baixo custo de motocicletas e as facilidades em seu financiamento são os responsáveis pelo aumento expressivo dessa frota $^{(18)}$. Entre 1996 e 2009, o Brasil aumentou 800\% a taxa de mortalidade por acidente de motocicletas, com os maiores valores nos estados do Nordeste ${ }^{(19)}$. Um estudo realizado nessa região estimou que os anos potenciais de vida perdidos em ATT por motos é, em média, de 39,34 anos ${ }^{(6)}$, que reflete a gravidade desse tipo de acidente.

Indivíduos do sexo masculino, na faixa etária dos 20 aos 39 anos, são os mais envolvidos em ATT e em causas externas ${ }^{(2,10,18)}$. Dados da Pesquisa Nacional por Amostra de Domicílios (PNAD), realizado pelo IBGE no ano de 2008, apontou que os homens tem a probabilidade 2 vezes maior de se envolverem nesse tipo de acidente do que mulheres ${ }^{(20)}$. Uma das prováveis explicações para este achado é o fato de, culturalmente, o homem expor-se mais a situações de perigo, como o consumo de álcool e a condução de veículo automotor.

No ano de 2013, o Sistema de vigilância de fatores de risco e proteção para doenças crônicas por inquérito telefônico (VIGITEL) e a Pesquisa Nacional de Saúde (PNS) entrevistaram, respectivamente, 52.929 e 60.202 pessoas com idade a partir de 18 anos. Em ambas as pesquisas registrou-se a média de $25 \%$ de motoristas adultos de carro ou moto que dirigiram logo depois de beber, sendo essa proporção significativamente maior no sexo masculino e para indivíduos jovens de 18 a 29 anos ${ }^{(21)}$.

A morbidade por ATT é um fenômeno de grande magnitude e alta complexidade ${ }^{(6)}$, em que vários fatores podem interferir nos acidentes e variar durante os anos. Os fatores de risco para ATT incluem dirigir sob o efeito de bebidas alcoólicas, estresse, fadiga, tonturas, excesso de velocidade, falta de uso de equipamentos de segurança (principalmente cinto de segurança e capacete), manutenção inadequada dos veículos e infraestrutura deficiente do sistema viário ${ }^{(22)}$. 
No presente estudo, observou-se maior proporção de internações por ATT no triênio 2011-2013, mostrando pequena redução no triênio 2014-2016. Todavia, uma análise sobre a tendência das internações decorrentes de ATT entre 2002 e 2012 , no Brasil, demonstrou aumento dessas taxas, com maior incremento (250\%) nas internações de motociclistas: 1,2 internações por 10 mil habitantes em 2002 e 4,2 internações por 10 mil habitantes em $2012^{(23)}$.

A proporção de acidentes envolvendo motocicletas pode variar consideravelmente entre as regiões brasileiras. Em estados da região Sudeste registram-se menores taxas quando comparadas ao presente estudo ${ }^{(24,25)}$.

A estratégia adotada no Brasil para redução de ATT a partir de 2008 estabeleceu alcoolemia zero e penalidades mais severas ao condutor que dirigir sob a influência do álcool ${ }^{(18)}$. Em 2012, a nova Lei Seca, a Lei n ${ }^{0} 12.760 / 2012$, aperfeiçoou o marco legislatório e possivelmente contribuiu para a redução do ato de dirigir sob efeito de álcool, ao estabelecer outras provas testemunhais e ampliar as sanções ao infrator ${ }^{(20)}$. Porém, não há consenso na literatura quanto à redução imediata nos índices de ATT após a implantação da Lei Seca.

Entre outras ações que podem ter influenciado na redução da proporção de IATT no triênio 2014-2016 no estado do Rio Grande do Norte, além das fiscalizações e ações de promoção à saúde, pode ser citada a implantação do Projeto Vida no Trânsito. Implantou-se o projeto em 2013 na capital do estado, contando com ações intersetoriais, dentre elas, ações socioeducativas e de redução dos fatores de riscos para os acidentes.

A região Nordeste figura entre as regiões brasileiras que registra as maiores taxas de internação por ATT, sendo os motociclistas as vítimas com maior permanência hospitalar e gastos ${ }^{(26,27)}$. Esses dados corroboram a presente pesquisa, que mostrou elevados gastos com internação por ATT comparado ao montante de gastos com internação no estado. Apesar das internações por ATT por si só demonstrarem alta proporção de gastos, é importante destacar que os danos causados às vítimas são físicos e psicológicos, e não se limitam aos gastos hospitalares, conforme elevados valores demonstrados em serviços com outros profissionais no Brasil ${ }^{(17)}$.

As proporções de internação por ATT nas regiões de saúde do Rio Grande do Norte, no qual é possível observar a elevada proporção de IATT por moto nas regiões de Santa Cruz, Caicó e Pau dos Ferros, demonstram a intensificação do trânsito e dos veículos em cidades de pequeno porte como Santa Cruz, onde a motocicleta figura como um importante meio de transporte. Como agravante, nessas cidades há uma menor fiscalização sobre o uso de equipamentos de proteção e o uso de bebida alcóolica.

É notório considerar que uma importante parcela da população vitimada em ATT pode não necessitar de internação( ${ }^{(2)}$, o que revela a magnitude e a urgência em ações acerca dos ATT como medidas preventivas intersetoriais ${ }^{(6)}$, com ações de promoção e prevenção focadas em usuários de motocicletas ${ }^{(18,27)}$. Apesar das medidas já existentes para a prevenção dos ATT, sugere-se maior envolvimento dos setores de saúde, de educação e de assistência para que a população tenha maior benefício em prevenção, em proteção e em recuperação da saúde.

O delineamento do presente estudo não confere informações detalhadas acerca das causas dos ATT e informações individuais, como aqueles que eventualmente permaneçam internados por longos períodos participando da proporção de gastos de mais de um ano, representando um limite do estudo. Contudo, a natureza dos dados e os corretos procedimentos de pesquisa adotados fortalecem a confiabilidade nos resultados apresentados.

\section{CONCLUSÃO}

No estado do Rio Grande do Norte encontrou-se elevada proporção de internações por acidentes com motocicletas em relação ao total de internações por acidentes de transporte terrestre, acometendo principalmente jovens do sexo masculino, com aumento de gastos nessas internações no triênio 2011-2013.

\section{REFERÊNCIAS}

1. Mello Jorge MHP, Oliveira LR. Análise epidemiológica das causas externas em unidades de urgência e emergência em Cuiabá / Mato Grosso. Rev Bras Epidemiol. 2008;11(3):420-30.

2. Golias ARC, Caetano R. Acidentes entre motocicletas: análise dos casos ocorridos no estado do Paraná entre julho de 2010 e junho de 2011. Ciênc Saúde Coletiva. 2013;18(5):1235-46.

3. Martins CBG, Jorge MHPM. Óbitos por causas externas em Cuiabá, 0 a 24 anos : perfil das vítimas e famílias segundo a intencionalidade. Rev Bras Epidemiol. 2013;16(2):454-68.

4. Rocha GS, Schor N. Acidentes de motocicleta no município de Rio Branco: caracterização e tendências. Ciênc Saúde Coletiva. 2013;18(3):721-31.

5. Martins ET, Boing AF, Peres, MA. Mortalidade por acidentes de motocicleta no Brasil: análise de tendência temporal , 1996-2009. Rev Saúde Pública. 2013;47(5):931-41. 
6. Almeida APB, Lima MLC, Oliveira FJM Júnior, Abath MB, Lima MLLT. Anos potenciais de vida perdidos por acidentes de transporte no Estado de Pernambuco, Brasil, em 2007. Epidemiol Serv Saúde. 2013;22(2):235-42.

7. Legay LF, Santos AS, Lovisi GM, Aguiar JSD, Borges JC, et al. Acidentes de transporte envolvendo motocicletas : perfil epidemiológico das vítimas de três capitais de estados brasileiros, 2007. Epidemiol Serv Saúde. 2012;21(2):283-92.

8. Mascarenhas MDM, Souto RMCV, Malta DC, Silva MMAD, Lima CMD, Montenegro MDMS. Características de motociclistas envolvidos em acidentes de transporte atendidos em serviços públicos de urgência e emergência. Ciênc Saúde Coletiva. 2016;21(12):3661-71.

9. Marín-león L, Belon AP, Barros MBA, Almeida SDM, Restitutti MC. Tendência dos acidentes de trânsito em Campinas , São Paulo, Brasil: importância crescente dos motociclistas. Cad Saúde Pública. 2012;28(1):39-51.

10. Sant'Anna FL, Andrade SM, Sant'Anna FHM, Liberatti CLB. Acidentes com motociclistas: comparação entre os anos 1998 e 2010. Londrina, PR, Brasil. Rev Saúde Pública. 2013;47(3):607-15.

11. Mesquita M Filho, Silva FM, Veiga VT. Acidentes de trânsito ocorridos antes e depois da legislação restritiva ao consumo de bebidas alcoólicas. Rev Méd Minas Gerais. 2012;22(3):259-64.

12. Morais OL Neto, Montenegro MDMS, Monteiro RA, Siqueira JB Júnior, Silva MMA, Lima CM, et al. Mortalidade por acidentes de transporte terrestre no brasil na última década: tendência e aglomerados de risco. Ciênc Saúde Coletiva. 2010;17(9):2223-36.

13. Ministério da Saúde (BR). Portaria GM/MS no 737, de 16/05/2001 - publicada no DOU nº 96, Seção 1E de 18/05/2011, que institui a Política Nacional de Redução da Morbimortalidade por Acidentes e Violências. 2a ed. Brasília: Ministério da Saúde; 2005. (Série E - Legislação de Saúde).

14. Ministério da Saúde (BR), Secretaria de Vigilância em Saúde (BR), Departamento de Análise de Situação em Saúde. Projeto Vigilância de acidentes e violências rede serviço sentinela de acidentes e violências. Brasília: Ministério da Saúde; 2006.

15. Instituto Brasileiro de Geografia e Estatística - IBGE. Brasil. Rio Grande do Norte. [acesso em 2017 Set 29]. Disponível em: https://cidades.ibge.gov.br/brasil/rn/panorama

16. Governo do Estado do Rio Grande do Norte, Secretaria de Estado da Saúde Pública. Plano Estadual de Saúde PAS 2016-2019. Natal: SESAP; 2016 [acesso em 2017 Set 29]. Disponível em: http://adcon.rn.gov.br/ACERVO/sesap/DOC/ DOC000000000142450.PDF

17. Andrade SSCA, Jorge MHPM. Internações hospitalares por lesões decorrentes de acidente de transporte terrestre. Epidemiol Serv Saúde. 2017;26(1):31-8.

18. Almeida RLF, Bezerra JG Filho, Braga JU, Magalhães FB, Macedo MCM, Silva KA. Via, homem e veículo: fatores de risco associados à gravidade dos acidentes de trânsito. Rev Saúde Pública. 2013;47(4):718-31.

19. Martins ET, Boing AF, Peres MA. Motorcycle accident mortality time trends in Brazil, 1996-2009. Rev Saúde Pública. 2013;47(5):931-41.

20. Malta DC, Mascarenhas MDM, Bernal RTI, Silva MMA, Pereira CA, Minayo MCS, Morais OL Neto. Análise das ocorrências das lesões no trânsito e fatores relacionados segundo resultados da Pesquisa Nacional por Amostra de Domicílios (PNAD) - Brasil, 2008. Ciênc Saúde Coletiva. 2008;16(9):3679-87.

21. Malta DC, Bernal RTI, Mascarenhas MDM, Silva MMA, Szwarcwald CL, Morais OL Neto. Consumo de bebidas alcoólicas e direção de veículos nas capitais brasileiras e no Distrito Federal, segundo dois inquéritos nacionais de saúde. Rev Bras Epidemiol. 2015; 18 (Supl 2):214-23.

22. Organización Panamericana de la Salud. Informe sobre el estado de la seguridade vial en la Región de las Américas. Washington: OPAS; 2009.

23. Ministério da Saúde (BR), Secretaria de Vigilância em Saúde, Departamento de Análise de Situação de Saúde. Saúde Brasil 2012: uma análise da situação de saúde e dos 40 anos do Programa Nacional de Imunizações. Brasília: Ministério da Saúde; 2013.

24. Abreu AMM, Jomar RT, Thomaz RGF, Guimarães RM, Lima JMB, Figueiró RFS. Impacto da Lei Seca na mortalidade por acidentes de trânsito. Rev Enferm UERJ, 2012;20(1):21-6.

25. Mesquita M Filho, Silva FM, Veiga VT. Acidentes de trânsito ocorridos antes e depois da legislação restritiva ao consumo de bebidas alcoólicas. Rev Méd Minas Gerais. 2012;22(3):259-64. 
26. Silva MMA, Morais OL Neto, Lima CMD, Malta DC, Silva BD Jr. Projeto Vida no Trânsito-2010-2012: uma contribuição para uma década de ações de segurança no trânsito 2011-2020 no Brasil. Epidemiol Serv Saúde. 2013;22(3):531-6.

27. Garden CRB, Souza Javhd, Lenardt MH, Pesck RM, Seima MD, Borges PKDO. Caracterização de idosos vítimas de acidentes por causas externas. Cogitare Enferm. 2014;19(3):506-13.

\section{Endereço para correspondência:}

Isabelle Ribeiro Barbosa

Universidade Federal do Rio Grande do Norte - UFRN

Faculdade de Ciências da Saúde do Trairi

Rua Vila Trairi, S/N

Bairro: Centro

CEP: 59200-000 - Santa Cruz - RN - Brasil

E-mail: isabelleribeiro@oi.com.br

\section{Endereço do primeiro autor:}

Ulicélia Nascimento de Azevedo

Universidade Federal do Rio Grande do Norte - UFRN

Faculdade de Ciências da Saúde do Trairi

Rua Vila Trairi, S/N

Bairro: Centro

CEP: 59200-000 - Santa Cruz - RN - Brasil

E-mail: ulicelia@yahoo.com.br 